Open Access

\title{
Comparison of Kompetitive Allele Specific PCR (KASP) and genotyping by sequencing (GBS) for quality control analysis in maize
}

Berhanu Tadesse Ertiro ${ }^{1 *}$, Veronica Ogugo ${ }^{2}$, Mosisa Worku², Biswanath Das², Michael Olsen², Maryke Labuschagne ${ }^{3}$ and Kassa Semagn ${ }^{2}$

\begin{abstract}
Background: Quality control (QC) analysis is an important component in maize breeding and seed systems. Genotyping by next-generation sequencing (GBS) is an emerging method of SNP genotyping, which is being increasingly adopted for discovery applications, but its suitability for QC analysis has not been explored. The objectives of our study were 1) to evaluate the level of genetic purity and identity among two to nine seed sources of 16 inbred lines using 191 Kompetitive Allele Specific PCR (KASP) and 257,268 GBS markers, and 2) compare the correlation between the KASP-based low and the GBS-based high marker density on QC analysis.

Results: Genetic purity within each seed source varied from 49 to $100 \%$ for KASP and from 74 to $100 \%$ for GBS. All except one of the inbred lines obtained from CIMMYT showed 98 to $100 \%$ homogeneity irrespective of the marker type. On the contrary, only 16 and $21 \%$ of the samples obtained from EIAR and partners showed $\geq 95 \%$ purity for KASP and GBS, respectively. The genetic distance among multiple sources of the same line designation varied from 0.000 to 0.295 for KASP and from 0.004 to 0.230 for GBS. Five lines from CIMMYT showed $\leq 0.05$ distance among multiple sources of the same line designation; the remaining eleven inbred lines, including two from CIMMYT and nine from Ethiopia showed higher than expected genetic distances for two or more seed sources. The correlation between the 191 KASP and 257,268 GBS markers was 0.88 for purity and 0.93 for identity. A reduction in the number of GBS markers to 1,343 decreased the correlation coefficient only by 0.03 .
\end{abstract}

Conclusions: Our results clearly showed high discrepancy both in genetic purity and identity by the origin of the seed sources (institutions) irrespective of the type of genotyping platform and number of markers used for analyses. Although there were some numerical differences between KASP and GBS, the overall conclusions reached from both methods was basically similar, which clearly suggests that smaller subset of preselected and high quality markers are sufficient for QC analysis that can easily be done using low marker density genotyping platforms, such as KASP. Results from this study would be highly relevant for plant breeders and seed system specialists.

Keywords: Genetic purity, Genetic identity, High marker density, Low marker density, Quality analysis, Single nucleotide polymorphism

\footnotetext{
* Correspondence: btadde@yahoo.com

${ }^{1}$ Ethiopian Institute of Agricultural Research (EIAR), Bako National Maize

Research Center, Bako, West Shoa, Oromia, Ethiopia

Full list of author information is available at the end of the article
} 


\section{Background}

In sub Saharan Africa (SSA), maize (Zea mays L.) is a staple food for more than 300 million people and is commonly grown by small-scale and resource-poor farmers in rural areas [1]. In Ethiopia, maize is the largest and most productive crop. In the 14 years period between 2000 and 2013, total annual production ranged from 2.7 to 6.7 million tons (http://faostat3.fao.org). During the same period, (i) maize yield in the country doubled from $1.6 \mathrm{t} \mathrm{ha}^{-1}$ in 2000 to $3.2 \mathrm{t} \mathrm{ha}^{-1}$ in 2013; and (ii) after eight years of erratic production, grain yield showed a rapid increase since 2007 [2]. However, productivity still remains far below the potential due to several factors, including periodic drought, high incidence of biotic stresses (diseases, insectpests and parasitic weeds), poor soil fertility, scarcity of irrigation water, and inadequate farmer access to affordable quality seeds and fertilizers.

The formal state maize breeding program of Ethiopia was established in the early 1950's and was instrumental in the development, evaluation and recommendation of adapted open pollinated varieties (OPVs). After nearly four decades, the breeding program released its first top cross hybrid, BH140, in 1988 [3] Subsequently, several high yielding and stress tolerant OPVs and hybrids adapted to different agro-ecologies have been released. These hybrid varieties, in conjunction with recent hybrids from private seed companies have significantly contributed to the current sharp increase in maize production in the country.

Initial adoption of hybrids by resource poor farmers was very slow for a number of reasons, including (i) high cost of hybrid seed relative to OPVs (especially as seed of OPVs can be recycled) (ii) limited or no access to improved hybrid seed in some regions; (iii) inadequate knowledge on agronomic management; (iv) insufficient seed companies and seed regulations in the country; (v) inadequate seed production infrastructure; and (vi) high cost of fertilizers [4]. The demand for hybrid seed gradually increased in Ethiopia as a result of changes in government policy including, but not limited to, the establishment of several local seed companies and the launching of a national extension program by government and non-governmental organizations (NGOs), such as Sasakawa Global 2000. The extension programs have made significant contribution in awareness creation of hybrid seed through field demonstration and providing technical support on hybrid maize grain production. Such rapid growth in hybrid adoption, however, brought a major concern on the quality of hybrid seed sold to resource poor farmers. Farmers reported high level of mixture of plants in their fields, and low yield in a given area. Despite the increased number of actors in the seed production and marketing venture, a vibrant national seed regulatory body to undertake effective seed quality assurance, including seed inspection and certification has been missing. Routine inspection of the initial parental seed (breeder, pre-basic and basic seed) produced by different actors in the seed value chain is critical and often done by inspecting production fields at vegetative and flowering stages. However, inspection of seed production fields based on a limited number of morphological and agronomic traits is time consuming, laborious, expensive, and at times can lead to inaccurate conclusions. Verification of seed lots and seed production fields can be effectively improved through the use of quality control (QC) genotyping using molecular markers.

Inbred lines are assumed to be genetically pure and possess all the genetic qualities that a breeder has selected for. Small changes in allele frequencies may occur during seed regeneration and maintenance breeding, and possible contamination with seeds or pollen of other samples [5, 6] Significant changes in the genetic makeup of a line may affect performance, and in the worst scenario result in distribution of wrong hybrids. Maintenance of inbred line genetic purity (homogeneity) and confirmation of the genetic identity of the same inbred line maintained at different locations are therefore important QC functions in maize breeding programs [7]. Several authors $[5,8-16]$ have reported the presence of a wide range of genetic differences among different seed sources of the same line designation. A high degree of differences among different seed sources of the same inbred line was also reported for some CIMMYT lines [7]. Thus breeding programs and seed distribution organizations must monitor the quality of seed increase and line maintenance processes using reliable tools to maintain the genetic homogeneity and identity of their key germplasm.

Single nucleotide polymorphic (SNP) markers have emerged as powerful tools for many genetic applications, including germplasm characterization (genetic diversity, genetic relationship, and population structure), QC analysis (genetic identity, genetic purity, and parentage verification), quantitative trait loci (QTL) mapping, allele mining, marker-assisted backcrossing, marker-assisted recurrent selection, and genomic selection. SNP data can be obtained using one of the numerous uniplex or multiplex SNP genotyping platforms that combine a variety of chemistries, detection methods, and reaction formats. Kompetitive Allele Specific PCR (KASP) is a uniplex SNP genotyping platform, and has developed into a global benchmark technology. CIMMYT uses a subset of 100 to 200 SNPs for routine QC genotyping using the KASP platform at LGC Genomics in the UK. The subset of SNPs for QC genotyping were selected out of the 1536 Illumina GoldenGate random chip using the following criteria: (1) ease of scoring with unambiguous separation of the two homozygous and heterozygous genotypes; (2) a minor allele frequency (MAF) and 
polymorphism information content (PIC) of at least 0.20 and 0.25 , respectively; (3) good distribution across chromosomes based on physical position, and (4) good polymorphism across a wide range of mapping populations. The genotyping cost for KASP depends on the number of data points ( 1 data point $=1$ sample genotyped by 1 $\mathrm{SNP}$ ) and data turnaround, which is 4 to 6 weeks for normal turnaround and 2 to 3 weeks for rapid turnaround. The current KASP genotyping cost for normal and rapid data turnaround ranges from US $\$ 0.064$ to US $\$ 0.242$ and from US $\$ 0.100$ to US $\$ 0.360$, respectively [7]. Genotyping by sequencing (GBS) [17] is an alternative method that could be used in generating high density genotype data at a genotyping and allele calling cost ranging from $\$ 18$ to $\$ 38$ per sample, depending on the level of multiplex (http://www.biotech.cornell.edu/brc/ genomic-diversity-facility/services/gbs-project-design-

and-optimization). To our knowledge, however, the correlation between GBS and KASP markers for QC analyses is not known. The objectives of our study were to 1) evaluate the level of genetic purity within each inbred line and understand genetic identity among different seed sources of the same line designation; and 2) compare the correlation between the KASP-based low density and the GBS-based high density information for QC analysis.

\section{Methods}

\section{Sample preparation and genotyping}

A total of 80 samples from 16 inbred lines, which are parental lines of eight popular Ethiopian hybrids (BH140, BH540, BHQP542, BH543, BHQPY545, BH660, BH670, and BH661), were used in this study (Table 1). Each inbred line was represented by from two to nine seed sources collected from the maize breeding program of the Ethiopia Institute of Agricultural Research (EIAR), seed companies in Ethiopia, the Ethiopian Institute of Biodiversity Conservation (IBC) (the national gene bank), and the International Maize and Wheat Improvement Center (CIMMYT) (Table 1). The seed samples from IBC were used as reference for older EIAR inbred lines, while the seeds obtained from CIMMYT were used to compare with the corresponding line designation maintained by EIAR and partners in Ethiopia. Seedlings were raised on plastic trays at the Biosciences eastern and central Africa (BecA) hub screen-house in Nairobi, Kenya. A single leaf from each of ten plants per sample were piled together, the tips trimmed off and approximately equal amount of leaf segment cut at once to make a bulk, and transferred into $1.2 \mathrm{~mL}$ strip tubes that contained two 4-mm stainless steel grinding balls (Spex CetriPrep, USA). Genomic DNA was extracted using a modified version of the CIMMYT high throughput mini-prep Cetyl Trimethyl Ammonium Bromide (CTAB) method as described elsewhere [18].
This extraction protocol has longer steps but provides good quality DNA for different purposes, including GBS that involves restriction digestion. DNA concentration was measured using the Quant-iT ${ }^{\mathrm{Tm}}$ PicoGreen $^{\circ}$ dsDNA assay kit (Invitrogen ${ }^{\mathrm{nw}}$, Paisley, UK) and the Tecan Infinite F200 Pro Plate Reader (Grödig, Austria), and normalized to $50 \mathrm{ng} / \mu \mathrm{L}$. For GBS, the quality of the extracted DNA was checked by digesting $250 \mathrm{ng}$ of the genomic DNA from 8 randomly selected samples with 3.6 units of ApeKI restriction enzyme (New England Biolabs, Boston, USA) at $75{ }^{\circ} \mathrm{C}$ for three hours. DNA samples were shipped to both LGC Genomics (http://www.lgcgroup.com) and the Genomic Diversity facility at Cornell University (http:// www.biotech.cornell.edu/brc/genomic-diversity-facility). Samples were genotyped with 200 SNPs (Additional file 1) prioritized by CIMMYT for QC genotyping using KASP genotyping platform [7]. The same DNA samples were also genotyped using GBS as described by Elshire and colleagues [17]. GBS data was generated by the Genomic Diversity Facility, Cornell University using ApeKI as restriction enzyme and 96-plex multiplexing.

\section{Data analyses}

The raw allele calls received from LGC Genomics consisted of several unassigned SNP calls. To minimize the unassigned calls, the KASP data was rescored using Kluster caller software from LGC Genomics. Nine SNPs were excluded either due to large missing data points or ambiguity in clearly discriminating the homozygous and heterogeneous genotypes so analyses were conducted using 191 of the 200 SNPs. Since GBS generates a high percentage of un-called genotypes, the missing data was imputed by the Institute of Genomic Diversity (IGD), Cornell University using an algorithm that searches for the closest neighbor in small SNP windows across the maize database [19]. Imputed data for 955,120 loci was received, but the majority of the GBS markers were monomorphic. The imputed GBS data was filtered using a minor allele frequency (MAF) of 0.05 in TASSEL version 4.3.2 software [20], yielding 257,268 polymorphic SNPs (26.9 \% of the initial loci) for further analyses (Table 2). The proportion of missing data after filtering with a MAF of 0.05 varied between 2.6 and 14.4, and the overall average across the 80 samples was $7.3 \%$.

Eight datasets were used for all statistical analyses. Dataset 1 and dataset 2 consisted of the 191 KASP SNPs and the 257,268 GBS markers after filtering using a MAF of 0.05 , respectively. Six additional input files were later created for correlation analyses: (a) dataset 3 consisted of a subset of 100 out of 191 SNPs routinely used by CIMMYT for QC genotyping; and (b) data set 4 to dataset 8 were created from dataset 2 using a MAF of $0.10,0.20,0.30,0.40$ and 0.50 . For QC analysis, the best SNPs would be those which amplify the two alleles 
Table 1 Proportion of homogeneity (purity) for 80 samples from 16 inbred lines using 191 KASP and 257,268 GBS markers

\begin{tabular}{|c|c|c|c|c|c|}
\hline \multirow[t]{2}{*}{ No } & \multirow[t]{2}{*}{ Name } & \multirow[t]{2}{*}{ Seed class } & \multirow[t]{2}{*}{ Source } & \multicolumn{2}{|c|}{ Homogeneity (\%) } \\
\hline & & & & KASP & GBS \\
\hline 1 & SC22_AR & Basic & AgriCEFT & 65 & 79 \\
\hline 2 & SC22_AR09 & Pre-basic & AARC & 59 & 76 \\
\hline 3 & SC22_BARC2011k & Basic & BARC & 66 & 80 \\
\hline 4 & SC22_BARC2012k & Basic & BARC & 69 & 81 \\
\hline 5 & SC22_EB & Nucleolus & BNMR & 79 & 86 \\
\hline 6 & SC22_GB & Gene bank & Gene Bank & 73 & 82 \\
\hline 7 & SC22_HD04/05 & Pre-basic & HARC & 63 & 79 \\
\hline 8 & SC22_I11B & Pre-basic & BNMR & 67 & 81 \\
\hline 9 & SC22_I12B & Pre-basic & BNMR & 64 & 80 \\
\hline 10 & 124-b(113)_AR09 & Pre-basic & AARC & 88 & 88 \\
\hline 11 & 124-b(113)_ASE & Basic & ASE & 76 & 83 \\
\hline 12 & 124-b(113)_BARC2010k & Basic & BARC & 82 & 85 \\
\hline 13 & 124-b(113)_BARC2011k & Basic & BARC & 84 & 85 \\
\hline 14 & 124-b(113)_EB & Nucleolus & BNMR & 88 & 89 \\
\hline 15 & 124-b(113)_GB & Gene bank & Gene Bank & 89 & 89 \\
\hline 16 & 124-b(113)_HD & Pre-basic & HARC & 75 & 82 \\
\hline 17 & 124-b(113)_HD04/05 & Pre-basic & HARC & 69 & 80 \\
\hline 18 & 124-b(113)_I12K & pre-basic & BNMR & 83 & 85 \\
\hline 19 & 124-b(109)_EB & Nucleolus & BNMR & 68 & 81 \\
\hline 20 & 124-b(109)_HD04/05 & Pre-basic & HARC & 58 & 74 \\
\hline 21 & 124-b(109)_I11B & Pre-basic & BNMR & 59 & 74 \\
\hline 22 & 124-b(109)_I13B & Pre-basic & BNMR & 56 & 74 \\
\hline 23 & CML197 & CIMMYT & CIMMYT & 91 & 100 \\
\hline 24 & CML197_EB & Nucleolus & BNMR & 73 & 85 \\
\hline 25 & CML197_HD03/04 & Pre-basic & HARC & 69 & 84 \\
\hline 26 & CML197_I10K & Pre-basic & BNMR & 69 & 84 \\
\hline 27 & CML197_I11K & Pre-basic & BNMR & 72 & 85 \\
\hline 28 & CML197_I12K & Pre-basic & BNMR & 67 & 85 \\
\hline 29 & CML312 & CIMMYT & CIMMYT & 100 & 100 \\
\hline 30 & CML312_EB & Nucleolus & BNMR & 99 & 100 \\
\hline 31 & A7033_AR02/03E.C & Pre-basic & AgriCEFT & 61 & 79 \\
\hline 32 & A7033_AR08 & Pre-basic & AARC & 63 & 80 \\
\hline 33 & A7033_EB & Nucleolus & BNMR & 75 & 85 \\
\hline 34 & A7033_GB & Gene bank & Gene Bank & 76 & 84 \\
\hline 35 & A7033_HD & Pre-basic & HARC & 61 & 82 \\
\hline 36 & A7033_I10K & Pre-basic & BNMR & 62 & 80 \\
\hline 37 & A7033_111K & Pre-basic & BNMR & 65 & 81 \\
\hline 38 & A7033_selected & Breeder seed & BNMR & 70 & 84 \\
\hline 39 & F7215_AR02/03E.C & Pre-basic & AgriCEFT & 63 & 77 \\
\hline 40 & F7215_AR08 & Pre-basic & AARC & 69 & 78 \\
\hline 41 & F7215_EB & Nucleolus & BNMR & 77 & 82 \\
\hline 42 & F7215_GB & Gene bank & Gene Bank & 64 & 78 \\
\hline 43 & F7215_HD04/05 & Pre-basic & HARC & 67 & 77 \\
\hline
\end{tabular}


Table 1 Proportion of homogeneity (purity) for 80 samples from 16 inbred lines using 191 KASP and 257,268 GBS markers (Continued)

\begin{tabular}{|c|c|c|c|c|c|}
\hline 44 & F7215_I11K & Pre-basic & BNMR & 65 & 77 \\
\hline 45 & F7215_I12K & Pre-basic & BNMR & 67 & 78 \\
\hline 46 & F7215_selected & breeder seed & BNMR & 70 & 80 \\
\hline 47 & 142-1-e_AR02/03E.C & Basic & AgriCEFT & 82 & 83 \\
\hline 48 & 142-1-e_EB & Nucleolus & BNMR & 89 & 86 \\
\hline 49 & 142-1-e_GB & Gene bank & Gene Bank & 89 & 87 \\
\hline 50 & 142-1-e_I10K & Pre-basic & BNMR & 85 & 85 \\
\hline 51 & 142-1-e_I12K & Pre-basic & BNMR & 87 & 84 \\
\hline 52 & CML202 & CIMMYT & CIMMYT & 99 & 100 \\
\hline 53 & CML202_12K & Pre-basic & BNMR & 99 & 99 \\
\hline 54 & CML202_EB & Nucleolus & BNMR & 99 & 99 \\
\hline 55 & CML202_HD04/05 & Pre-basic & HARC & 96 & 99 \\
\hline 56 & CML202_I11K & pre-basic & BNMR & 92 & 98 \\
\hline 57 & CML395 & CIMMYT & CIMMYT & 100 & 100 \\
\hline 58 & CML395_12K & Pre-basic & BNMR & 64 & 81 \\
\hline 59 & CML395_EB & Nucleolus & BNMR & 49 & 77 \\
\hline 60 & CML395_HD & Pre-basic & HARC & 60 & 81 \\
\hline 61 & CML395_I11K & Pre-basic & BNMR & 59 & 81 \\
\hline 62 & 144-7-b_108K & Pre-basic & BNMR & 80 & 84 \\
\hline 63 & 144-7-b_109k & Pre-basic & BNMR & 79 & 83 \\
\hline 64 & 144-7-b_I10k & Pre-basic & BNMR & 81 & 84 \\
\hline 65 & CML144 & CIMMYT & CIMMYT & 99 & 100 \\
\hline 66 & CML144_EB & Nucleus & BNMR & 98 & 99 \\
\hline 67 & CML144_I11B & Pre-basic & BNMR & 98 & 98 \\
\hline 68 & CML144_I13B & Pre-basic & BNMR & 96 & 97 \\
\hline 69 & CML159_EB & Nucleolus & BNMR & 98 & 99 \\
\hline 70 & CML159_I09K & Pre-basic & BNMR & 93 & 97 \\
\hline 71 & CML159_I13B & Pre-basic & BNMR & 90 & 96 \\
\hline 72 & CML176_EB & Nucleolus & BNMR & 87 & 94 \\
\hline 73 & CML176_I12K & Pre-basic & BNMR & 83 & 94 \\
\hline 74 & CML161 & CIMMYT & CIMMYT & 100 & 100 \\
\hline 75 & CML161_I12B & Pre-basic & BNMR & 99 & 100 \\
\hline 76 & CML161_I13B & Pre-basic & BNMR & 100 & 100 \\
\hline 77 & CML165 & CIMMYT & CIMMYT & 98 & 100 \\
\hline 78 & CML165_EB & Nucleolus & BNMR & 95 & 98 \\
\hline 79 & CML165_I11B & Pre-basic & BNMR & 98 & 99 \\
\hline 80 & CML165_I12B & Pre-basic & BNMR & 88 & 93 \\
\hline
\end{tabular}

equally (i.e., each with a frequency of 0.50 ). The proportion of heterogeneity (the number of markers that were not homozygous due to mixture of two homozygous genotypes or residual heterozygosity) in each sample was calculated from all datasets using TASSEL version 4.3.2. Genetic purity was calculated from all datasets in Excel as the difference between 100-h, where $\mathrm{h}$ refers to heterogeneity in percentage obtained from TASSEL. For all eight datasets, genetic distance was calculated between each pair of samples using the identity by descent (IBS) method implemented in TASSEL. Dendrograms were constructed from the genetic distance matrices of both dataset 1 and dataset 2 using the Unweighted Pair Group Method with Arithmetic Mean (UPGMA) algorithm 
Table 2 Summary of the number of KASP and GBS markers used in the present study

\begin{tabular}{lll}
\hline Chromosome & GBS & KASP \\
\hline 1 & 40,666 & 26 \\
2 & 31,600 & 21 \\
3 & 30,120 & 19 \\
4 & 23,977 & 19 \\
5 & 29,656 & 19 \\
6 & 20,880 & 17 \\
7 & 21,084 & 16 \\
8 & 21,651 & 19 \\
9 & 19,886 & 19 \\
10 & 17,748 & 16 \\
Total & 257,268 & 191 \\
\hline
\end{tabular}

implemented in molecular evolutionary genetics analysis (MEGA), version 6 [21]. Mantel tests [22] were used to compute the correlation between the genetic distance matrices derived from all eight datasets using NTSYS-pc (numerical taxonomy and multivariate analysis system), version 2.11 [23]. Pearson correlation coefficients between genetic purity values obtained for all eight datasets were calculated using MINITAB v14.

\section{Results and discussion}

\section{Genetic purity (homogeneity)}

We first computed the proportion of homozygous SNPs on each of the 80 samples as an estimate of genetic purity or homogeneity. The results were highly variable across samples, with homogeneity varying from 49 to $100 \%$ for KASP and from 74 to $100 \%$ for GBS (Table 1; Fig. 1). The overall average homogeneity across all 80 samples was $79 \%$ for KASP and $87 \%$ for GBS. Most
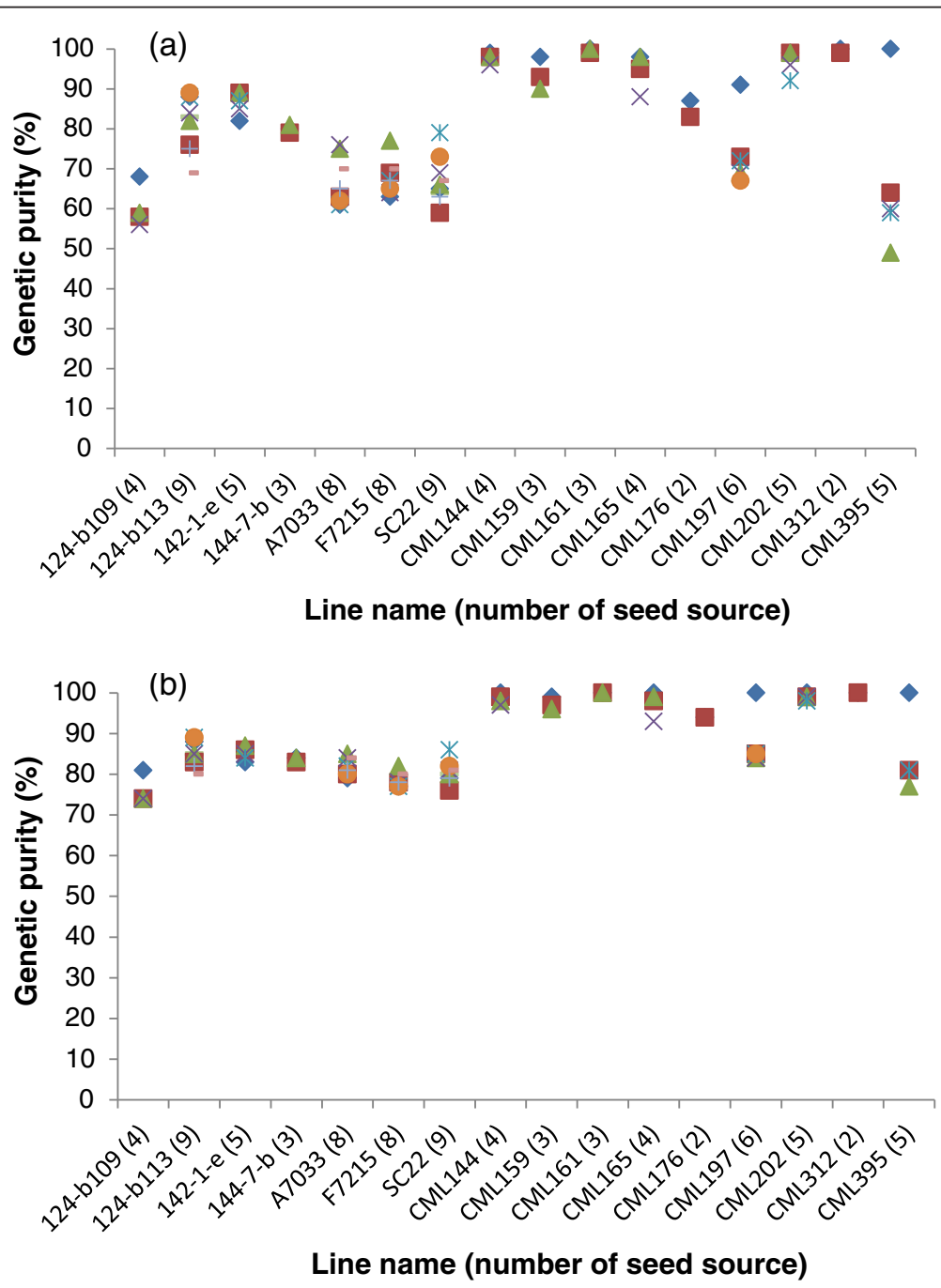

Fig. 1 Comparison of genetic purity of multiple seed sources of the 16 inbred lines using a 191 KASP and b 257,268 GBS. For each line designation, the number of seed sources is shown in the $x$-axis in bracket and with different shapes in the plot 
breeding programs now use inbred lines at $\mathrm{F}_{4}$ or later generations, but previously, lines were often derived at earlier generations. An inbred line may be considered pure or homogenous if the proportion of heterozygous or heterogeneous loci does not exceed $5 \%$ [7]. Samples with substantially more than $5 \%$ heterogeneity (3.1\% due to residual heterozygosity in the founder plants plus $1.9 \%$ due to both genotyping error and genetic drift) for a given set of SNPs are likely to have been contaminated by pollen or seed of another genotype. In the present study, approximately $23 \%$ of the samples in KASP and $28 \%$ of the samples in GBS were considered genetically pure with $\leq 5 \%$ heterogeneity (Fig. 2). The majority of the samples (77\% in KASP and $72 \%$ in GBS) showed high proportion of heterogeneity that varied from 6 to $51 \%$ in KASP and from 6 to $26 \%$ in GBS.

Genetic purity among the multiple seed sources of each of the 16 inbred lines was compared to understand whether the high proportion of heterogeneity was specific to a few lines or common across most lines (Fig. 3). Genetic purity was consistently lower for all inbred lines developed by EIAR irrespective of their seed origin or marker density. The genetic purity of CIMMYT lines maintained at EIAR and partners was highly variable, with some showing much lower than the expected level of purity, while others had high level of purity. For example, the seed sources obtained from EIAR and partners for both CML395 and CML197 showed the lowest purity (49 and $73 \%$ for KASP and 77 and $85 \%$ for GBS, respectively), while CML312, CML202, CML144 and CML161 from EIAR and partners showed 96 to $100 \%$ purity regardless of sources and marker density (Table 1 ). All CML seed sources obtained from CIMMYT showed the highest purity, which varied between 98 and $100 \%$ for both KASP and GBS except CML197 that showed conflicting results for KASP (91 \%) and GBS (100\%).

Results above highlight two main points. First, the level of purity for most samples originating from EIAR and partners was low because most inbred lines sampled from these sources were early generation inbred lines used as parents for old commercial hybrids in Ethiopia. Prior to the release of hybrids, maize farmers in the country used to grow OPVs for several reasons, including the relatively higher cost of imported hybrid seeds and higher input recommendation for hybrids as compared to OPVs [24]. In addition, the source germplasm which was available for line development at the time was unimproved and intolerant to inbreeding depression. To cope with these challenges, breeders of the time opted to develop and release hybrids using early generation parental inbred lines [3]. Although this strategy favors cheaper seed production and hybrids derived from such early generation parental lines out-yielded OPVs, generally they remain inferior in uniformity as compared to hybrids developed from fixed lines.

Second, the level of purity from the present study clearly agrees with the stage of inbreeding and our field observation on lack of uniformity for most of the older EIAR inbred lines. Complaints from growers on the unexpectedly high level of variability under farmers' field conditions is associated with a combination of genetic reasons, handling of early generation inbred lines and inadequate seed inspection and quality assurance system for hybrid seed production in the country. However, mislabeling, pollen contamination and seed admixture are some of the other major reasons that might have contributed for the variation observed under the farmers' field.

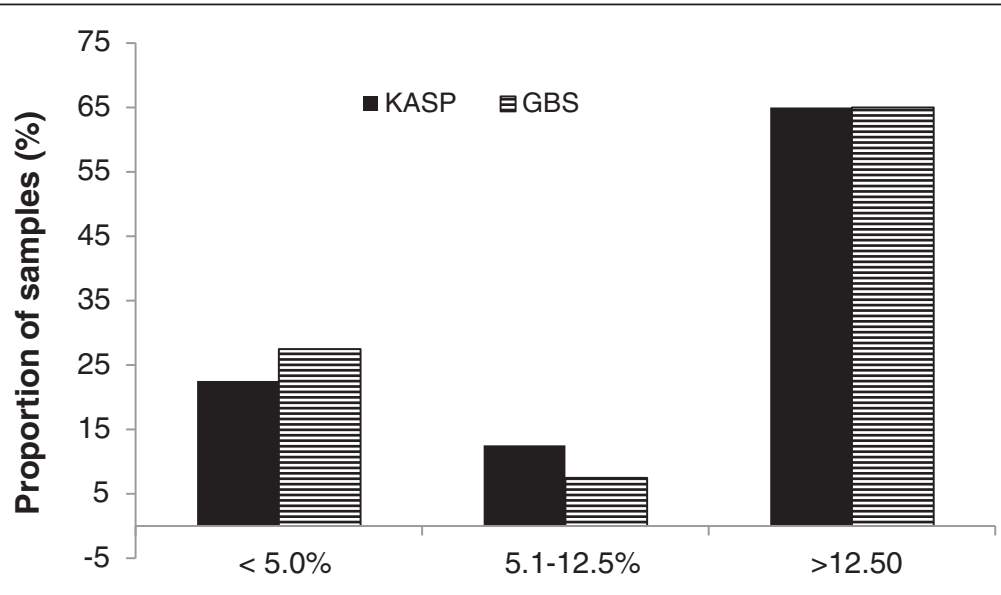

Heterogeneity (\%)

Fig. 2 Summary of the heterogeneity of 80 seed sources from 16 inbred lines based on 191 KASP and 257,268 GBS markers. See Table 1 for homogeneity values of each marker 

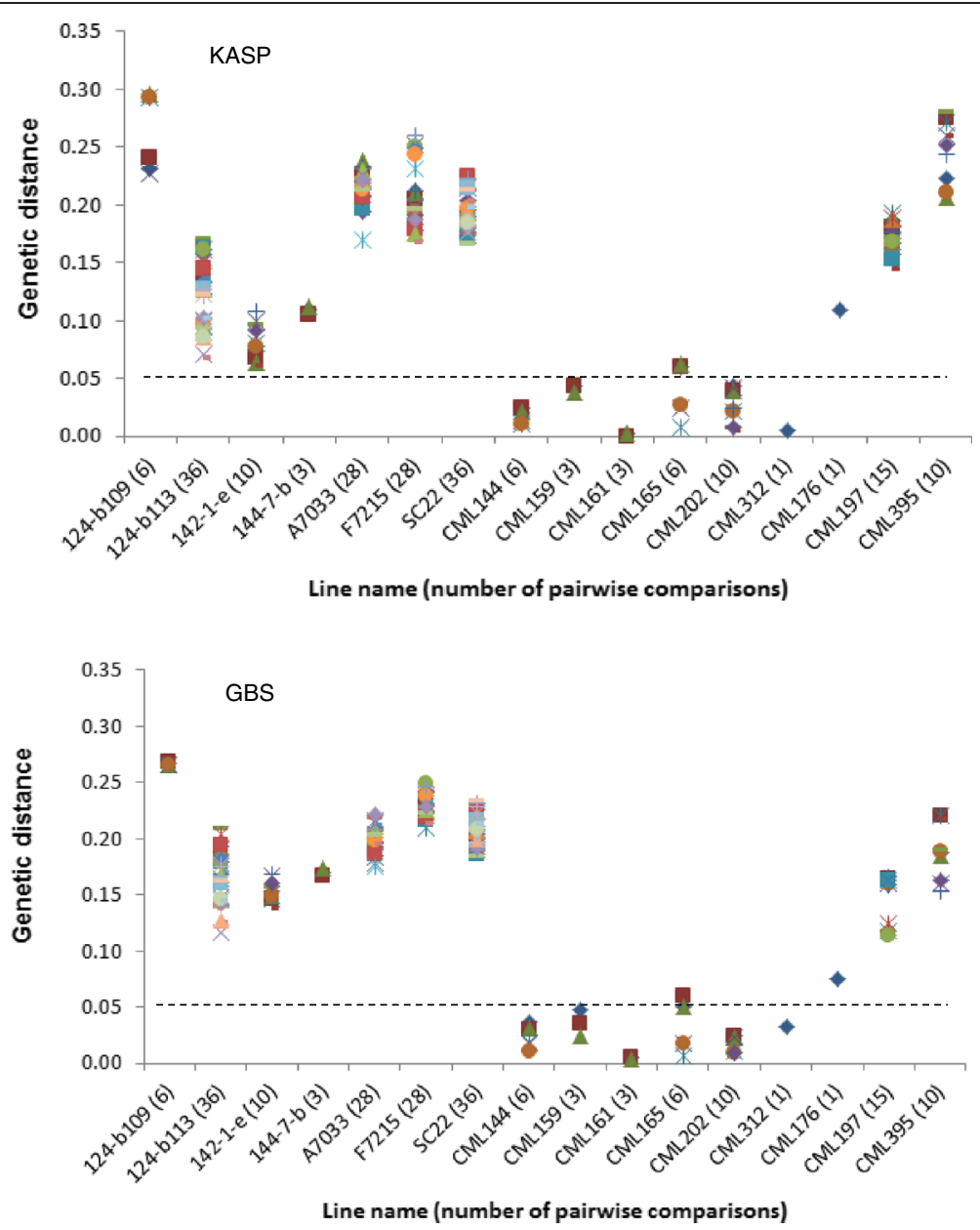

Fig. 3 Summary of pairwise comparisons of genetic distance among multiple sources of the same line designation genotyped with 191 KASP and 257,268 GBS markers. For each line designation, the number of seed sources is shown in the $x$-axis in bracket and with different shapes in the plot

\section{Genetic identity}

Multiple seed sources of a given inbred line developed after four generations of inbreeding are expected to be genetically identical or nearly so with a maximum allelic difference of $<5 \%$ [7]. In the present study, genetic distance among the different seed sources of the same line designation varied from 0.000 to 0.295 for KASP and from 0.004 to 0.230 for GBS. As shown in Fig. 3, the genetic distance among all pairewise comparisons of seed sources for five CMLs (CML144, CML159, CML161, CML202 and CML312) was $<0.05$. The remaining eleven inbred lines, including four CMLs and all seven lines from EIAR, showed higher proportion of mismatch for two or more seed sources. The dendrograms in both Figs. 4 and 5 also clearly show the extent of genetic differences among multiple seed sources of the same line designation. Importantly, dendrograms from both KASP and GBS datasets have grouped different seed sources of the same line designation together except 124-b (109) EB. The grouping of all sources of the same line designation together is an indication that all sources of the same line were derived from the same origin, but most of them have diverged significantly for several reasons, including residual heterozygosity, seed or pollen contamination, genetic drift and the method of line maintenance. Labeling error is the most likely explanation for one of the samples of 124-b (109) EB mis-grouping from the other sources (Figs. 4 and 5). The effect of method and frequency of maintenance on genetic structure of heterozygous individuals is hastened by natural and artificial selection, which entails the elimination of individuals carrying undesirable alleles $[5,11,13]$. To maintain allele frequencies in early generation inbred lines, it is compulsory to raise large population sizes in the sibbing blocks in order to avoid genetic drift. In standard line maintenance, however, the breeders would most likely be using few plants which can affect the identity of early generation lines amongst sources due to drift and selection bias. Because of this 


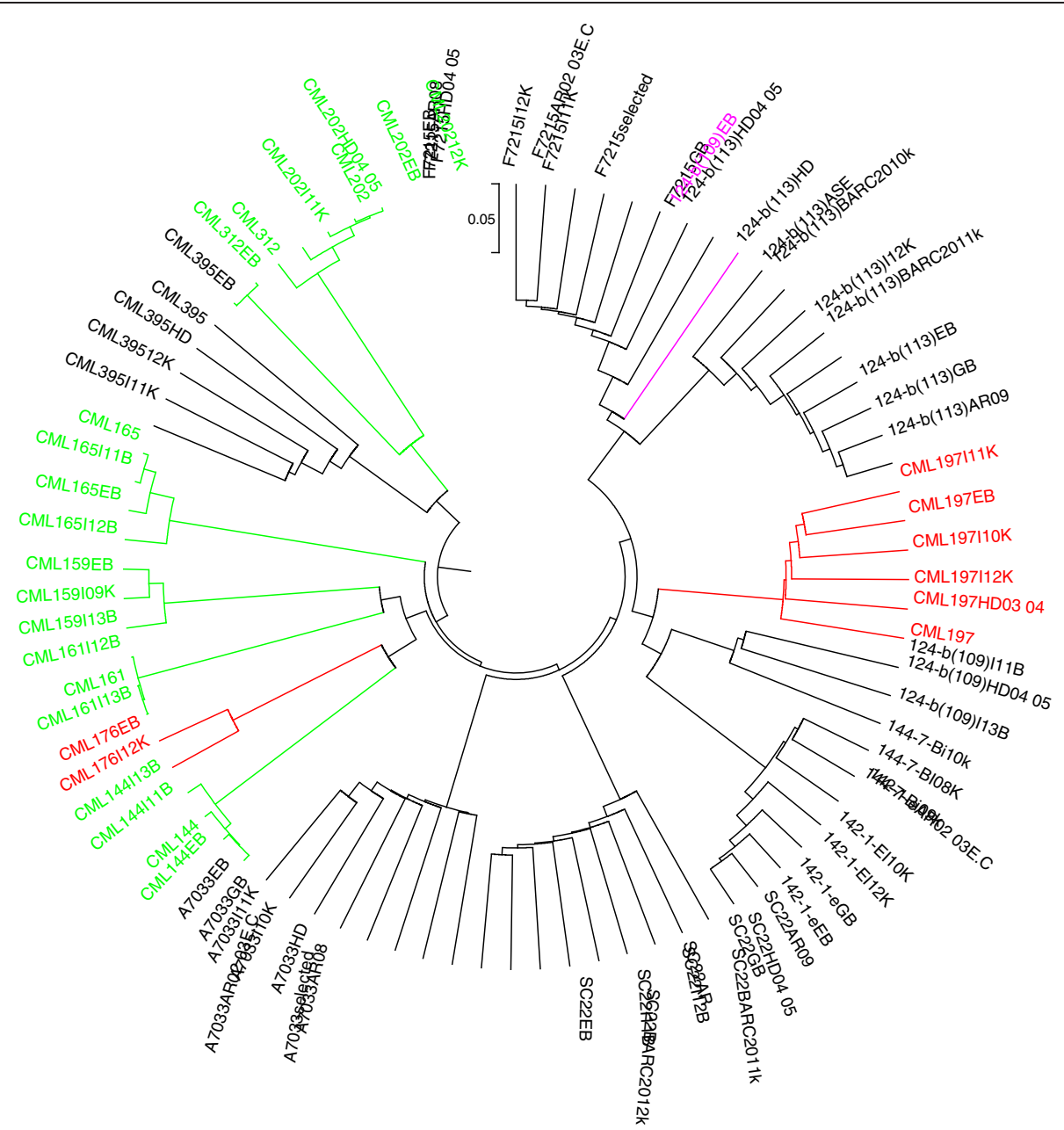

Fig. 4 UPGMA dendrogram of 80 samples from 16 inbred lines based on genetic distance matrix computed from 191 KASP SNPS

practical difficulty, EIAR breeders have been incrementing pre-basic/basic seed of all early generation inbred lines only in isolated blocks in order to minimize genetic drift. This method allows for frequent inspection of seed production fields along with rigorous rouging when off-type plants are observed; and has helped to maintain early generation lines for more than two decades enabling farmers to access true-to-type hybrid seed every season. Reliable seed production has contributed to the sharp increase in maize productivity in the country for food security. However, when seed companies started to produce their own pre-basic/basic seed in different locations, the supply of consistent hybrid seed to farmers become a challenge. Significant variation of performance of various seed lots of the same hybrid designation was observed, likely due to differences in parental line maintenance methods.

\section{Correlation between low and high marker density}

Semagn et al. [7] prioritized a subset of about 100 to 200 SNPs for routine QC analysis using KASP genotyping platform. In order to understand the relationship between marker types and densities using KASP or GBS markers for estimating genetic purity and identity, we conducted correlation analyses by creating several subsets of data (Fig. 6). The correlation between the subset of 100 and 191 KASP SNPs recommended for QC analysis by Semagn et al. [7] was 0.95 for identity and 0.99 for purity. When the 100 KASP markers were compared with the entire 257,268 GBS markers, the correlation coefficients were 0.82 for identity and 0.90 for purity. The increase in KASP markers from 100 to 191 increased the correlation with GBS by only 0.03 for purity and 0.06 for identity. The KASP and GBS markers showed some discrepancy in terms of numerical values when heterogeneity exceeded $12.5 \%$, with $61.3 \%$ of the samples showing 12.5 to $25 \%$ heterogeneity in GBS and $43.8 \%$ of the samples showing $>25 \%$ heterogeneity in KASP (Fig. 3). However, the conclusions reached in assigning lines into genetically pure or not were highly similar. Given the low accuracy of GBS in correctly calling 


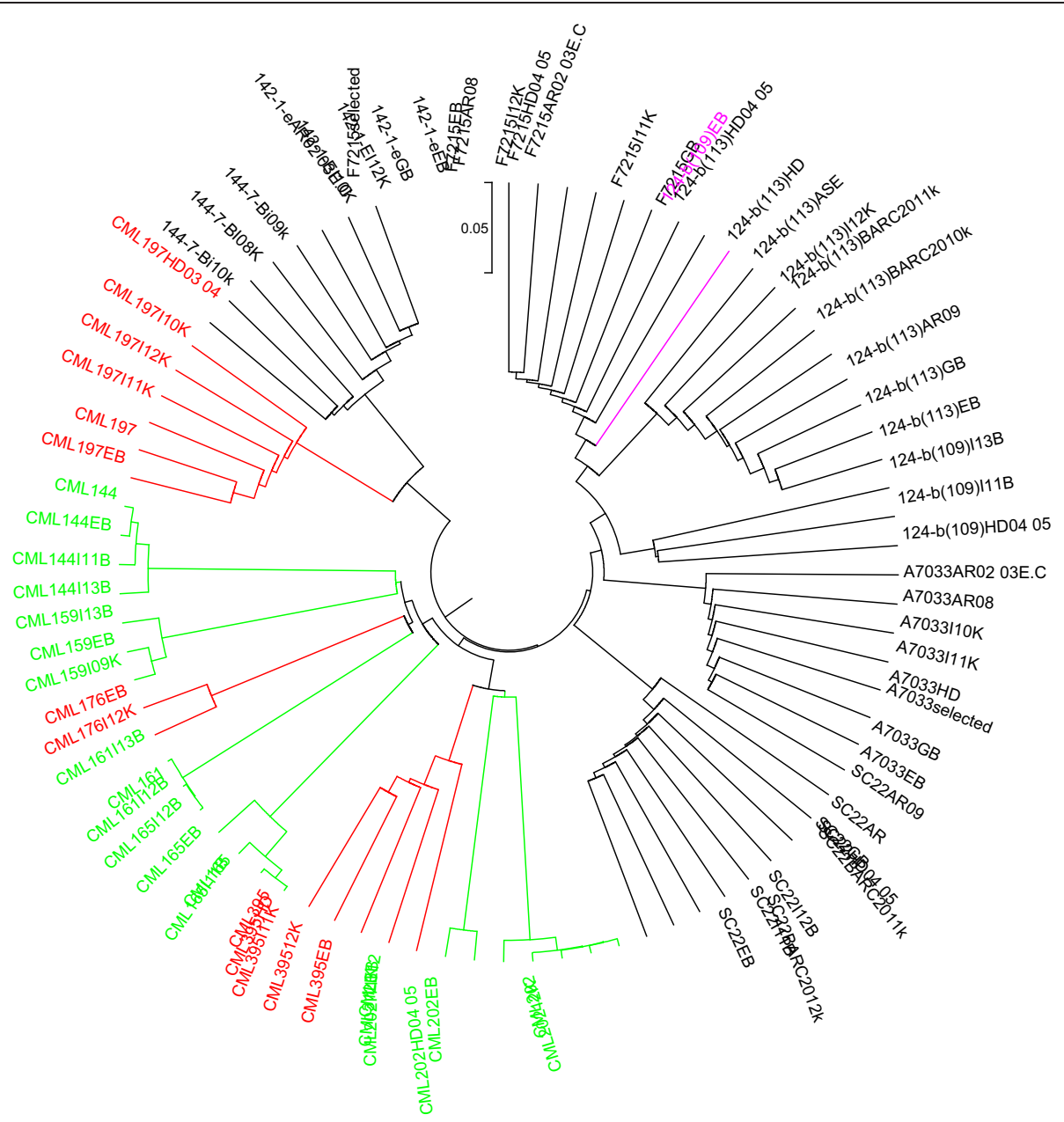

Fig. 5 UPGMA dendrogram for 80 seed sources from 16 inbred lines based on genetic distance matrix computed from 257,268 GBS markers

highly heterogeneous and heterozygous germplasm and some of the issues associated in imputing GBS calls, such discrepancies may be expected. A reduction in GBS markers from 257,268 to 1,343 did not impact correlation values for the various pairwise comparisons (Fig. 6). Therefore, the effect of marker density for routine QC analysis seems relatively minor. It is concluded that 100 to 200 SNP markers routinely used by CIMMYT for QC genotyping are sufficient for genetic purity and identity purposes. The use of high density GBS markers for QC analysis, at least at present, does not add value to the process for different reasons, including longer data turnaround time and lower accuracy in correctly calling alleles in highly heterogeneous and heterozygous germplasm.

\section{Implication of the QC results and recommendations}

Overall, most seed sources from CIMMYT were considered genetically pure, which was not the case for the majority of samples originating from EIAR. Such results, however, are expected for inbred lines developed with only a few generations of inbreeding. One of the major objectives of maize breeders in Ethiopia during the 1980s was to develop maize germplasm that performed better than OPVs in order to address the outcry over food insecurity in the country. During that time, breeders had limited access to diverse maize germplasm for new pedigree starts, there were no private companies involved in maize breeding and/or seed multiplication and marketing in the country, and there were no clear seed regulations governing quality control and assurance. Breeders were pressured to release improved hybrids which could outperform widely grown OPVs. They used early generation inbred lines to develop and release hybrids, and maintained these lines in isolation increases without losing their identity. This contributed to sharp growth of maize production in the country. Currently, Ethiopia has a relatively well established seed law for QC/QA using morpho-agronomic traits. This can be strengthened by incorporating modern molecular tools. Maize breeders in the country also have better access to a wide range of germplasm for new pedigree starts from different national programs in Africa, CIMMYT, and the 


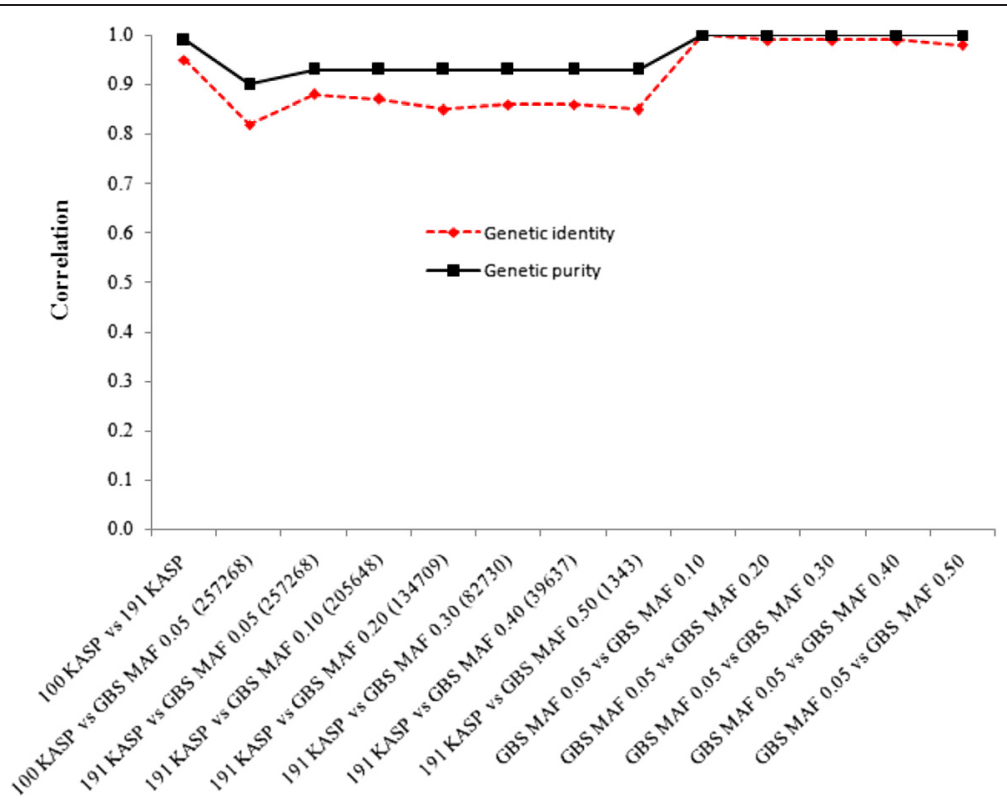

Fig. 6 Correlation coefficients between different number of KASP and GBS markers for genetic purity and identity estimated from 80 samples

International Institute for Tropical Agriculture (IITA). They are now developing inbred lines after four or later generations of selfing and more recently released hybrids developed using inbred lines with higher genetic purity. This will assist to maintain homogeneous parental inbred lines in the next generation hybrids in Ethiopia.

The use of parental inbred lines with high heterogeneity in breeding programs can have multifold negative effects, including the use of wrong donors in new breeding starts for line development, hybrid formation as well as genetic and molecular studies. Genetic purity of a given parental line also has major impact on the production and distribution of certified seed to farmers. Failure to undertake regular quality control analysis could result in generation and dissemination of incorrect products to the end user. Maize breeders commonly exchange seed of the most widely used inbred lines; therefore, unexpected level of genetic heterogeneity in a given seed lot or high mismatch across different seed sources of a given inbred line can quickly spread across different programs with consequent negative effects. Obtaining seed from a reliable source and undertaking routine quality control, will be useful for minimizing errors associated with purity and identity. Our results showed that a subset of 100 to 200 SNP (KASP) markers would be sufficient for routine $\mathrm{QC}$ analysis. In cases where there is no reference genotype data for inbred lines that will be used for comparison purposes, it is recommended to grow out multiple sources of different sources of the same line designation in nurseries, generate SNP data, compare SNP data with field notes, and discard those sources that show deviation from expectation in terms of purity and identity. CIMMYT has carried out this exercise to resolve genetic purity and identity issues from 280 seed sources involving 40 inbred lines and strongly recommends this regular activity for other breeders (M. Worku, personal communication). The information presented in this paper would be highly useful for maize breeders that are involved in new pedigree start, developing populations for QTL mapping and marker-assisted breeding, and the seed companies.

\section{Conclusions}

Our results clearly showed high discrepancy both in genetic purity and identity by the origin of the seed sources (institutions) irrespective of the type of genotyping platform and number of markers used for analyses. Overall, most seed sources from CIMMYT were considered genetically pure, which was not the case for the majority of samples originated from EIAR. One of the reasons for such discrepancy in genetic purity and identity was the level of inbreeding prior to releasing the parental lines for hybrid formation. Although there were some differences between KASP and GBS results, the overall conclusions reached from both methods was basically similar, which clearly suggests that smaller subset of preselected high quality markers are sufficient for QC analysis that can easily be done using low marker density genotyping platforms, such as KASP. GBS data would be highly useful for establishing reference marker database at the time of releasing an inbred parental line for used in heterotic grouping and planning hybrid combinations and new pedigree 
starts. Results from this study would be highly relevant for plant breeders and seed system specialists.

\section{Additional file}

\section{Additional file 1: Summary of the 200 KASP SNPs used for} genotyping. (XLSX $17 \mathrm{~kb}$ )

\section{Abbreviations}

CIMMYT: International maize and wheat improvement center; CTAB: Cetyl trimethyl ammonium bromide; EIAR: Ethiopian Institute of Agricultural Research; GBS: Genotyping by sequencing IBS, identity by descent; IGD: Institute of genomic diversity; KASP: Kompetitive Allele Specific PCR MAF, minor allele frequency; MEGA: Molecular evolutionary genetics analysis; NGOs: Non-governmental organizations; OPVs: Open-pollinated varieties; QC: Quality control analysis; SNP: Single nucleotide polymorphism; SSA: Sub Saharan Africa; UPGMA: Unweighted pair group method with arithmetic mean.

\section{Competing interests}

The authors declare no competing financial interests.

\section{Authors' contributions}

BTE was responsible for data generation, analyses and drafting the manuscript; $\mathrm{VO}, \mathrm{MW}, \mathrm{BD}, \mathrm{MO}, \mathrm{ML}$ and $\mathrm{KS}$ made their contribution in data analyses and editing the manuscript. All authors have read and approved the manuscript.

\section{Authors' information}

BTE is a PhD student; VO is a research assistant at CIMMYT; MW, BD, MO and KS are research scientists at CIMMYT; ML is a professor at the University of Free State, South Africa.

\section{Acknowledgment}

This work was financed by African Bioscience Challenge Fellowship fund (ABCF) managed by Bioscience eastern and central Africa (BecA) hub.

\section{Author details}

${ }^{1}$ Ethiopian Institute of Agricultural Research (EIAR), Bako National Maize Research Center, Bako, West Shoa, Oromia, Ethiopia. ${ }^{2}$ International Maize and Wheat Improvement Center (CIMMYT), P. O. Box 1041 Village Market, 00621 Nairobi, Kenya. ${ }^{3}$ Department of Plant Sciences, University of Free State, Bloemfontein, South Africa.

Received: 2 September 2015 Accepted: 31 October 2015

Published online: 06 November 2015

\section{References}

1. Shiferaw B, Prasanna BM, Hellin J, Banziger M. Crops that feed the world 6. Past successes and future challenges to the role played by maize in global food security. Food Security. 2011;3(3):307-27.

2. Abate T, Shiferaw B, Menkir A, Wegary D, Kebede $Y$, Tesfaye K, et al. Factors that transformed maize productivity in Ethiopia. Food Security. 2015;7(5):965-81.

3. Tolessa B, Gobezayehu T, Worku M, Desalegne Y, Mulatu K, Bogale G. Genetic Improvement of maize in Ethiopia. In: Proceedings of the first national maize workshop of Ethiopia: 5-7 May 1993; Addis Ababa. Ethiopia: IAR/CIMMYT; 1993. p. 13-22.

4. Semagn K, Magorokosho C, Ogugo V, Makumbi D, Warburton M. Genetic relationships and structure among open-pollinated maize varieties adapted to eastern and southern Africa using microsatellite markers. Mol Breed. 2014:34(3):1423-35.

5. Heckenberger M, Bohn M, Ziegle JS, Joe LK, Hauser JD, Hutton M, et al. Variation of DNA fingerprints among accessions within maize inbred lines and implications for identification of essentially derived varieties. I. Genetic and technical sources of variation in SSR data. Mol Breed. 2002;10(4):181-91.

6. Warburton ML, Setimela P, Franco J, Cordova H, Pixley K, Banziger M, et al. Toward a cost-effective fingerprinting methodology to distinguish maize open-pollinated varieties. Crop Sci. 2010;50(2):467-77.
7. Semagn K, Beyene Y, Makumbi D, Mugo S, Prasanna BM, Magorokosho C, et al. Quality control genotyping for assessment of genetic identity and purity in diverse tropical maize inbred lines. Theor Appl Genet. 2012;125(7):1487-501.

8. Jones DF. Heterosis resulting from degenerative changes. Genetics. 1945;30(6):527-42.

9. Schuler JF. Natural mutations in inbred lines of maize and their heterotic effect. I. comparison of parent, mutant and their F1 hybrid in a highly inbred background. Genetics. 1954;39(6):908-22.

10. Russell WA, Sprague GF, Penny LH. Mutations affecting quantitative characters in long-time inbred lines of Maize1. Crop Sci. 1963;3(2):175-8.

11. Fleming AA, Kozelnicky GM, Browne EB. Variations between stocks within long-time inbred lines of maize (Zea mays L.) 1. Crop Sci. 1964;4(3):291-5.

12. Russell WA, Vega UA. Genetic stability of quantitative characters in successive generations in maize inbred lines. Euphytica. 1973;22(1):172-80.

13. Gethi JG, Labate JA, Lamkey KR, Smith ME, Kresovich S. SSR variation in important U.S. maize inbred lines. Crop Sci. 2002;42(3):951-7.

14. Heckenberger $M$, Voort JR, Melchinger $A E_{t}$, Peleman J, Bohn M. Variation of DNA fingerprints among accessions within maize inbred lines and implications for identification of essentially derived varieties: II. Genetic and technical sources of variation in AFLP data and comparison with SSR data. Mol Breed. 2003;12(2):97-106

15. Revilla P, Abuin MC, Malvar RA, Soengas P, Ordas B, Ordas A. Genetic variation between Spanish and American versions of sweet corn inbred lines. Plant Breed. 2005;124(3):268-71.

16. Yan J, Shah T, Warburton ML, Buckler ES, McMullen MD, Crouch J. Genetic characterization and linkage disequilibrium estimation of a global maize collection using SNP markers. PLoSOne. 2009;4(12), e8451.

17. Elshire RJ, Glaubitz JC, Sun Q, Poland JA, Kawamoto K, Buckler ES, et al. A robust, simple genotyping-by-sequencing (GBS) approach for high diversity species. PLoS ONE. 2011;6(5):e19379.

18. Semagn K. Leaf Tissue Sampling and DNA Extraction Protocols. In: Besse P, editor. Molecular Plant Taxonomy: Methods and Protocols. vol. 1115. New York: Human Press; 2014. p. 53-67.

19. Romay M, Millard M, Glaubitz J, Peiffer J, Swarts K, Casstevens T, et al. Comprehensive genotyping of the USA national maize inbred seed bank. Genome Biol. 2013;14(6):R55.

20. Bradbury PJ, Zhang Z, Kroon DE, Casstevens TM, Ramdoss Y, Buckler ES. TASSEL: software for association mapping of complex traits in diverse samples. Bioinformatics. 2007;23(19):2633-5.

21. Tamura K, Stecher G, Peterson D, Filipski A, Kumar S. MEGA6: molecula evolutionary genetics analysis version 6.0. Mol Biol Evol. 2013;30(12):2725-9.

22. Mantel $\mathrm{N}$. The detection of disease clustering and a generalized regression approach. Cancer Res. 1967;27:209-20.

23. Rholf FJ. NTSYS-pc, Numerical Taxonomy and Multivariate Analysis System. New York: Exeter software; 1993.

24. Gebre T. Maize Technoloyg Adoption in Ethiopia: Experiences from the Sasakawa-Global 2000 Agriculture Program. In: Enhancing the Contribution of Maize to Food Security in Ethiopia. Addis Ababa: EIAR/CIMMYT; 2001. p. 232.

\section{Submit your next manuscript to BioMed Central and take full advantage of:}

- Convenient online submission

- Thorough peer review

- No space constraints or color figure charges

- Immediate publication on acceptance

- Inclusion in PubMed, CAS, Scopus and Google Scholar

- Research which is freely available for redistribution 